\title{
Identification of key miRNAs in the progression of hepatocellular carcinoma using an integrated bioinformatics approach
}

\author{
Qi Zheng ${ }^{1}$, Xiaoyong Wei ${ }^{\text {Corresp., }}{ }^{2}$, Jun Rao ${ }^{2}$, Cuncai Zhou ${ }^{2}$ \\ 1 Department of Oncology, Fuzhou First people's Hospital, Fuzhou, Jiangxi, China \\ 2 Department of Hepatobiliary Surgery, Jiangxi Cancer Hospital, Nanchang, Jiangxi, China \\ Corresponding Author: Xiaoyong Wei \\ Email address: weixiaoyong206@163.com
}

Backgroud: It has been shown that aberrant expression of microRNAs (miRNAs) and transcriptional factors (TFs) is tightly associated with the development of HCC. Therefore, in order to further understand the pathogenesis of HCC, it is necessary to systematically study the relationship between the expression of miRNAs, TF and genes. In this study, we aim to identify the potential transcriptomic markers of HCC through analyzing common microarray datasets, and further establish the differential co-expression network of miRNAs-TF-mRNA to screen for key miRNAs as candidate diagnostic markers for HCC.

Method: We first downloaded the mRNA and miRNA expression profiles of liver cancer from the GEO database. After pretreatment, we used a linear model to screen for differentially expressed genes (DEGs) and miRNAs. Further, we used weighed gene coexpression network analysis (WGCNA) to construct the differential gene co-expression network for these DEGs. Next, we identified mRNA modules significantly related to tumorigenesis in this network, and evaluated the relationship between mRNAs and TFs by TFBtools. Finally, the key miRNA was screened out in the mRNA-TF-miRNA ternary network constructed based on the target TF of differentially expressed miRNAs, and was further verified with external data set. Results: A total of 465 DEGs and 215 differentially expressed miRNAs were identified through differential genes expression analysis, and WGCNA was used to establish a co-expression network of DEGs. One module that closely related to tumorigenesis was obtained, including 33 genes. Next, a ternary network was constructed by selecting 256 pairs of mRNA-TF pairs and 100 pairs of miRNA-TF pairs. Network mining revealed that there were significant interactions between 18 mRNAs and 25 miRNAs. Finally, we used another independent data set to verify that miRNA hsamir-106b and hsa-mir-195 are good classifiers of HCC and might play key roles in the progression of HCC. Conclusion: Our data indicated that two miRNAs-hsa-mir-106b and hsa-mir-195 are identified as good classifiers of HCC.

Peer] reviewing PDF | (2019:10:42157:1:1:NEW 7 Mar 2020) 
1 Identification of key miRNAs in the progression of hepatocellular carcinoma

2 using an integrated bioinformatics approach

3

4 Qi Zheng ${ }^{1}$, Xiaoyong $\mathrm{Wei}^{2}$, Jun $\mathrm{Rao}^{2}$, Cuncai Zhou ${ }^{2}$

5

$6{ }^{1}$ Department of Oncology, Fuzhou First people's Hospital, Fuzhou, Jiangxi, China.

$7 \quad 2$ Department of Hepatobiliary Surgery, Jiangxi Cancer Hospital, Nanchang, Jiangxi, China

8

9 Corresponding author:

10 Xiao-yong $\mathrm{Wei}^{2}$

11 No. 519, East Beijing road, Nanchang City, Jiangxi Province, 330029, China.

12 Email address: weixiaoyong206@163.com 


\section{Abstract}

Backgroud: It has been shown that aberrant expression of microRNAs (miRNAs) and transcriptional factors (TFs) is tightly associated with the development of HCC. Therefore, in order to further understand the pathogenesis of HCC, it is necessary to systematically study the relationship between the expression of miRNAs, TF and genes. In this study, we aim to identify the potential transcriptomic markers of $\mathrm{HCC}$ through analyzing common microarray datasets, and further establish the differential co-expression network of miRNAs-TF-mRNA to screen for key miRNAs as candidate diagnostic markers for HCC.

Method: We first downloaded the mRNA and miRNA expression profiles of liver cancer from the GEO database. After pretreatment, we used a linear model to screen for differentially expressed genes (DEGs) and miRNAs. Further, we used weighed gene co-expression network analysis (WGCNA) to construct the differential gene co-expression network for these DEGs. Next, we identified mRNA modules significantly related to tumorigenesis in this network, and evaluated the relationship between mRNAs and TFs by TFBtools. Finally, the key miRNA was screened out in the mRNA-TF-miRNA ternary network constructed based on the target TF of differentially expressed miRNAs, and was further verified with external data set.

Results: A total of 465 DEGs and 215 differentially expressed miRNAs were identified through differential genes expression analysis, and WGCNA was used to establish a co-expression network of DEGs. One module that closely related to tumorigenesis was obtained, including 33 genes. Next, a ternary network was constructed by selecting 256 pairs of mRNA-TF pairs and 100 pairs of miRNA-TF pairs. Network mining revealed that there were significant interactions between 18 mRNAs and 25 miRNAs. Finally, we used another independent data set to verify that miRNA hsa-mir-106b and hsa-mir-195 are good classifiers of HCC and might play key roles in the progression of HCC.

Conclusion: Our data indicated that two miRNAs—-hsa-mir-106b and hsa-mir-195 are identified as good classifiers of HCC. 


\section{Introduction}

41 Hepatocellular carcinoma (HCC) is one of the most common cancers worldwide and the 42 second leading cause of global cancer-related death accounting for around $11 \%$ of all cancer deaths(J Ferlay et al., 2015). The pathogenesis of HCC, as well as the genes involved in this process is under intensive investigation. At present, the potentially curative options for HCC patients are radiofrequency ablation, liver transplantation and tumor resection. However, these options are only effective for the early stage of HCC(JC Nault et al., 2018). At the end stage of HCC, or when recurrence is occurred, the mean overall survival of patients are less than three months(L Yin et al., 2018). It is necessary to understand the molecular mechanism of HCC progression, in order to predict and control this disease as early as possible.

MicroRNAs (miRNAs) are small non-coding RNAs that are highly conserved between species and are associated with many important biological processes. MiRNAs usually exert their roles in post-translational regulation, by base-pairing to the 3'-untranslated regions (3'- 
65

association among genes in $\mathrm{HCC}$ development. Novel approaches of systematically studying the interaction network of protein-coding genes and miRNAs are still missing.

In this study, we aim to study the interactions of mRNA, miRNAs and TFs, to reveal key miRNAs that are involved in HCC development, and to explore novel indicators of HCC progression. By establishing a weighed gene co-expression network and analyzing mRNAmiRNA-TF interactions, we identified two miRNAs-hsa-mir-106b and hsa-mir-195, that share the most TFs with their interacting mRNAs. Gene set enrichment analysis (GSEA) revealed that they are both involved in metabolic process and immune response. Our data indicated that hsamir-106b and hsa-mir-195 are good classifiers of HCC and key players in the progression of liver cancer.

\section{Materials \& Methods}

The flowchart of identification of key mRNAs and mRNA-interacting miRNAs was shown in Fig. 1 and methods in detail are provided below.

\section{Dataset acquirement and processing}

Dataset acquirement:

We searched for HCC related datasets in GEO database (https://www.ncbi.nlm.nih.gov/geo/) and downloaded two gene profiles in Series Matrix format. One dataset named GSE36376 is an encoded gene expression profile data containing $211 \mathrm{HCC}$ samples and 243 adjacent non-tumor tissue samples, hybridized on Illumina HumanHT-12 V4.0 expression beadchip (platform NO. GPL10558)(HY Lim et al., 2013). The other dataset named GSE36915 is a miRNA profile hybridized on Illumina Human v2 MicroRNA expression beadchip (platform NO. GPL8179), containing 68 HCC samples and 21 non-tumor liver tissue samples(TC Shih et al., 2012).

Dataset processing: The dataset GSE36376 containing total of 47323 probes and the dataset GSE36915 
91 containing 1145 probes were interpreted according to the following procedures: 1 . In case that

92 one probe corresponded to one gene uniquely, directly transformed the name of this probe into

93 the corresponding gene symbol; 2 . In case that one probe corresponded to multiple genes, removed this probe; 3 . In case that multiple probes corresponded to one gene, transformed the mean value of these probes into the expression value of this gene. Altogether, we got the interpreted gene expression profile and miRNA profile containing 18076 and 1134 genes from the two datasets, respectively.

\section{Identification of differentially expressed genes (DEGs)}

In order to identify genes (including mRNA and miRNA) that play key roles in HCC progression, R package Limma was used for t-test analysis and $\mathrm{BH}$ method was used for controlling the false discovery rate (FDR) and calculating the fold change of expression value. Genes with the FDR value less than 0.01 and the $|\operatorname{logFC}|$ value more than 1 was considered significantly DEGs.

\section{Construction of weighted gene co-expression networks and identification of gene modules associated with HCC progression.}

WGCNA is a freely accessible R package for the construction of weighted gene coexpression networks(P Langfelder and S Horvath, 2008). The basis of WGCNA is to find gene modules that have co-expression relationship when they are used to distinguish a sample trait in two groups. The procedure of WGCNA is composed of two main steps, expression clustering analysis and phenotypic association analysis, as described previously. Three different ways can be selected to construct the network and identify modules according to different needs. In our study, the one-step function was used for network construction and detection of differentially expressed mRNA modules. 
114

115

116

117

118

119

120

121

122

123

124

125

126

127

128

129

130

131

132

133

134

135

136

137

138

\section{Prediction of mRNA and TF interaction}

Interaction of mRNA and TF were predicted using TFBstools. Gene modules obtained from the weighted gene co-expression networks were combined with TFs in JASPAR database, and further analyzed for potential binding sites of TFs on mRNA molecules. The sequence information of mRNAs was obtained from UCSC database, and two thousand base-pairs (2k bps) upstream from the transcription start site (TSS) were considered TF binding sites. The scores of mRNA-TF pairs were ranked and used for further analyzed.

\section{Prediction of miRNA-TF interaction}

To analysis the interaction of miRNA and TFs, predictive information of miRNA-TF pairs was downloaded from CricuitsDB database. The CricuitsDB database integrates genome-wide transcriptional and post-transcriptional regulatory networks based on bioinformatic gene sequence analysis, and it is designed to identify the complex feedforward loop regulatory relationships of human miRNA-TF (O Friard et al., 2010). To further enhance the reliability of the prediction, the miRNA-TF pairs were validated using two databases-TargetScan and TargetMiner (A Jeggari et al., 2012). The miRNA-TF pairs that were confirmed in at least one database and included in differentially expressed miRNAs (DEGs) were considered as differentially expressed miRNA-TF pairs.

\section{Construction of the mRNA-TF-miRNA triple network}

The mRNA-TF-miRNA triple interaction network was constructed as described previously (H Wang et al., 2017, L Zhao et al., 2018). Briefly, differentially expressed miRNA-TF pairs and mRNA-TF pairs (scoring at top 5\%) that screened out previously were integrated, and the interaction network was visualized using Cytoscape(P Shannon et al., 2003). We analyzed the topological properties of the regulatory networks with Cytoscape plugin and identified hubs.

\section{Identification of miRNA-mRNA pairs using hypergeometric distribution}

Interactions between miRNA and mRNAs in the triple network were evaluated based on 
139

$140 \mathrm{p}=1-\frac{\left(\begin{array}{l}\mathrm{k} \\ \mathrm{m}\end{array}\right)\left(\begin{array}{l}\mathrm{n}-\mathrm{k} \\ \mathrm{N}-\mathrm{m}\end{array}\right)}{\left(\begin{array}{l}\mathrm{n} \\ \mathrm{N}\end{array}\right)}$

$\mathrm{N}$ is the number of TFs, $\mathrm{m}$ is the number of mRNA-interacting TFs, $\mathrm{n}$ is the number of

miRNA-interacting TFs, $\mathrm{k}$ is the number of TFs that both interact with mRNAs and miRNAs,

and $p$ value is the significance of mRNA-miRNA interaction. $p<0.05$ is considered significant.

144

145

146

147

148

149

150

151

152

153

154

155

156

157

158

159

160

\section{Functional enrichment analysis}

Functional enrichment analysis of mRNA-interacting miRNAs were performed using R package clusterProfiler. GO-termed analysis of $\mathrm{CC}, \mathrm{MF}$ and $\mathrm{BP}$ were used to interpret the function of miRNA-mRNA pairs in HCC progression. Hierarchical network diagram of GO-term analysis was generated using binGO of Cytoscape.

\section{Validation of key miRNAs in independent expression profiles}

To test the classification capacity of selected miRNAs and mRNAs, an independent miRNA expression profile (GSE10694) was downloaded from GEO database and DEGs were screened out using $\mathrm{R}$ package Limma with the threshold of FDR $<0.05$. DEGs screened out from GSE10694 were further compared with miRNAs that were filtered out in the triple network. The miRNAs located in the intersection of these two miRNA expression profiles were considered key miRNAs that might drive HCC development.

\section{Results}

\section{Identification of DEGs from the expression profile of HCC}

In order to find differentially expressed mRNAs and miRNAs between HCC and non-tumor controls, two expression profiles - one mRNA profile GSE36376 and one miRNA profile 
161 GSE36915, were downloaded from GEO database, respectively. Totally, GSE36376 contained

16218076 mRNAs, while GSE36915 contained 1134 miRNAs. DEGs, including 465 differentially

163 expressed mRNAs between $211 \mathrm{HCC}$ tissues and 243 non-tumor tissues and 215 differentially

164 expressed miRNAs between 68 HCC tissues and 21 non-tumor tissues, were identified using t-

165 test analysis and FDR correction (listed in Table S1). Heatmaps of two profiles, as shown in Fig.

$1662 A-2 B$, respectively, indicated that these DEGs were good in distinguishing HCC samples and

167 non-tumor samples.

168

169

170

171

172

173

174

175

176

177

178

179

180

181

182

183

184

185

186

\section{Construction of weighed gene co-expression network}

Weighted gene co-expression networks are used to identify interesting gene modules, as well as the intramodular connectivity and gene significance based on the correlation of a gene expression profile with a sample trait. To explore the interaction of differentially expressed mRNAs, weighted gene co-expression network containing 465 mRNAs was constructed by WGCNA. The distributions of $\log (\mathrm{k})$ and $\log (\mathrm{p}(\mathrm{k}))$ coefficients and mean connectivity corresponding to thresholds of significance $(\beta)$ were computed by pickSoftThreshold and shown in Fig. 3A. Based on a scale-free topology criterion, $\beta=12$ was selected as the soft threshold power in the present study. To obtain the co-expression matrix, the minimal module size was set as 20 , and other parameters were set at default levels. The system clustering tree was constructed base on Pearson's coefficients of each gene (Fig. 3B-C). Therefore, genes were classified into three modules according to their correlation with traits. Among these, we found that the brown module eigengene (ME), which includes 33 mRNAs (listed in Table S2), displayed highest correlation index with traits $(\mathrm{R}=0.93, \mathrm{p}=2 \mathrm{e}-184$, Fig. 3D). Intramodular interaction of these 33 mRNAs were analyzed and shown in Fig. S1. Expression of these mRNAs was highly correlated with traits (Fig. 3E). Taken together, we found a co-expression gene hub containing 33 mRNAs that were differentially expressed in HCC samples compared with controls.

\section{Prediction of the mRNA-TF interactions}

To further explore potential interactions between mRNAs and TFs, we scored the binding of 
187

188

145 TFs (downloaded from JASPAR database, and listed in Table S3) to the 33 mRNAs identified above using TFBstools. The sequence information of mRNAs was downloaded from USCS database, and sequences in the range of $2 \mathrm{~kb}$ upstream from the TSS were picked up for predicting TF-binding sites. Top 5\% mRNA-TF pairs were selected for further analysis. Totally, 32 mRNAs, 85 mRNA-interacting TFs, and 256 mRNA-TF pairs were identified (listed in Table S4).

\section{Prediction of interactions of miRNAs and TFs}

The generation of miRNAs were regulated by TFs, while miRNAs were usually involved in post-translational regulation of TFs. Therefore, we predicted miRNA-interacting TFs by combining the information of three databases - CircuitsDB, TargetScan and TargetMiner. TFs that interact with differentially expressed miRNAs were obtained from CircuitsDB, and miRNATF pairs were subsequently validated in TargetScan and TargetMiner. Totally, 100 miRNA-TF pairs containing 28 miRNAs and 45 TFs were identified (Table S5).

\section{Generation of mRNA-TF-miRNA interaction network}

Based on the mRNA-TF pairs and miRNA-TF pairs identified above, a triple interaction network including mRNAs, TFs, and miRNAs was generated (Fig. 4). This network contained 356 edges, 32 mRNA hubs, 28 miRNA hubs and 127 TF hubs. We found that three TFsSTAT1, SRF and YY1 were special because they were simultaneously connected with both mRNAs and miRNAs. According to our results, these three TFs might play critical roles in the progression of HCC.

\section{Refining mRNA-miRNA pairs in the triple network}

To further identify core mRNA-miRNA interactions in the triple network, we used hypergeometric distribution method to evaluate the connectivity of mRNA-miRNA pairs. The degree of connectivity of mRNA-miRNA pairs were measured by the number of TFs they shared. Totally 100 mRNA-miRNA pairs, including 18 mRNAs and 25 miRNAs, were found to share one or more TFs (Table 1). According to our results, one miRNA interacts with several 
213 mRNAs while one mRNA interacts with several miRNAs.

214

215

216

217

218

219

220

221

222

223

224

225

226

227

228

229

230

231

232

233

234

235

236

237

238

\section{Functional enrichment analysis of mRNA-interacting miRNAs}

The complex interactions of mRNAs and miRNAs indicated the biological roles of miRNAs in the progression of HCC. To further study the biological functions of 25 miRNAs identified above, we performed GO-term enrichment analysis, which included cellular component (CC), molecular function (MF) and biological process (BP). The result of functional enrichment analysis indicated that these miRNAs were involved in the BPs such as negative regulation of vascular endothelial growth factor signaling pathway, response to iron on starvation, response to bile acid and urate homeostasis, and MFs such as adenylate cyclase binding, signaling pattern recognition receptor activity, and CCs such as collagen type VI trimer, cell cortex part (Fig. S2). Representative results of the GO-term analysis were shown in Fig. 5. GO-term analysis was further visualized by the hierarchical network diagram, which interpreted the weight and the hierarchical relationship of enriched GO-terms. The analysis results of two of the miRNAs- hsamiR-106b and hsa-miR-195 indicated that they are intensely involved in aminoglycan metabolic process, peptide cross-linking, positive regulation of cyclase activity and immune system process, immune response (Fig. 6). Taken together, results of functional enrichment analysis indicated that most of the mRNA-interacting miRNAs that identified in the network might play important role in the progression of HCC.

\section{Verification of expression of miRNAs in another independent case}

To further verify the reliability of the results, we downloaded an independent expression profile GSE10694 from GEO database. GSE10694 contains miRNA expression profiles in liver cancer tissues and corresponding non-tumor tissues of $78 \mathrm{HCC}$ cases, and in 10 normal liver tissues(W Li et al., 2008). Totally, 23 differentially expressed miRNAs were identified using Limma with the threshold of FDR $<0.05$. Among these miRNAs, miR-106b and hsa-miR-195 were selected twice in GSE10694 and GSE36915, successively. This result indicated that the two miRNAs are good classifiers of HCC and key players in the progression of liver cancer. On the 
239

240

241

\section{Discussion}

243

other hand, our results also confirmed that the method used to identify these miRNAs are reliable and can be applied in other cases.

1. In this study, we used deep and comprehensive bioinformatic analysis of three independent public datasets and identified a considerable number of mRNA-miRNA pairs. Moreover, our study utilized the central regulatory roles of TFs to construct a triple interaction network that can systematically interpret the relationship between miRNAs, TFs and mRNAs. TFs are key modulators of hepatic functions and are broadly involved in HCC development because of their DNA binding and transcriptional modulating activity. We found that 25 miRNAs and 18 mRNAs among these mRNA-miRNA pairs shared the most TFs and located in the central position of the miRNA-TF-mRNA triple interacting network. Therefore, we succeeded to predict that these mRNA-miRNA pairs might play central roles in the development of HCC and provide a novel way to explore differentially co-expression gene hubs and interpret their interactions. These co-expression gene hubs are valuable and can be used as diagnostic biomarkers for HCC, although more experimental verification of the results needed to be performed in the future.

2. Among the genes identified in the triple network, some have been previously connected to cancer progression in independent studies. For instance, signal transducer and activator of transcription 1 (STAT1), a transcriptional factor associated with type I and II interferon signaling, is considered to have anti-tumor activity in the HCC models. STAT1-deficient mice are more prone to tumor development than animals with wild-type, indicating the central role of STAT1 in HCC progression(V Shankaran et al., 2001). Serum response factor (SRF) is a transcription factor that in liver, can be activated by hepatitis B virus (HBV) and C virus (HCV). Constitutive activation of SRF in mice contributes to primary $\mathrm{HCC}$ formation(S Ohrnberger et al., 2015). In addition to TFs, other genes that are involved in immune 
response (MARCO)(H Sun et al., 2017), iron homeostasis regulation (HAMP)(F Ren et al., 2018), proteoglycans synthesis (ECM1(H Chen et al., 2011), DCN(Z Horvath et al., 2014)) have been reported to be associated with the progression or prognosis of HCC. Especially, two miRNAs-hsa-miR-106b and hsa-miR-195, which were verified as HCC classifiers in two independent profiles, have been also implicated in regulating HCC development. HsamiR-106b regulates the apoptosis and tumorigenesis of $\mathrm{HCC}$ via targeting $\mathrm{Zbtb} 7 \mathrm{a}(\mathrm{X}$ Liang et al., 2018), while hsa-miR-195 inhibits metastasis of HCC via targeting FGF2 and VEGFA(M Wang et al., 2015). Moreover, hsa-miR-195 has also been implicated to target PCMT1, a member of the type II class of protein carboxyl methyltransferase enzymes, thus regulating protein metabolism and facilitating the development of HCC(M Amer et al., 2014). Therefore, all these reports solidly confirm that the genes selected in this hub are closely correlated with the progression of HCC. However, because only three expression profiles have been used in this study, the reliability of results need to be confirmed in more independent cases.

\section{Conclusions}

In this study, we used an integrated bioinformatics approach to explore mRNA-miRNA-TF triple interactions that are highly associated with HCC development. Our data indicated that two miRNAs - hsa-mir-106b and hsa-mir-195 are identified as good classifiers of HCC. However, because miRNAs can directly bind to mRNAs and suppress their translational function, further studies should be performed to analysis the suppressive effect of miRNAs to mRNAs and TFs in this network. Moreover, before these results being applied in exploring clinical biomarkers or therapeutic targets, experimental studies should be performed to demonstrate the functional roles of these genes in different stages of HCC progression, respectively. 
292

293

294

295

296

297

298

299

300

301

302

303

304

305

306

307

308

309

310

311

312

313

314

315

316

317

318

319

320

321

322

323

324

325

326

327

328

\title{
Acknowledgments
}

\author{
The authors declare no conflict of interest.
}

\section{References}

Amer M, Elhefnawi M, El-Ahwany E, Awad AF, Gawad NA, Zada S, Tawab FM. 2014. Hsa-miR-195 targets PCMT1 in hepatocellular carcinoma that increases tumor life span. Tumour Biol 35; 11301-9. doi: 10.1007/s13277014-2445-4.

Bartel DP. 2004. MicroRNAs: genomics, biogenesis, mechanism, and function. Cell 116; 281-97. doi: 10.1016/S00928674(04)00045-5.

Boye A, Yang Y. 2014. Hepatic microRNA orchestra: a new diagnostic, prognostic and theranostic tool for hepatocarcinogenesis. Mini Rev Med Chem 14; 837-52. doi: 10.2174/138955751410141020151237.

Chen H, Jia WD, Li JS, Wang W, Xu GL, Ma JL, Ren WH, Ge YS, Yu JH, Liu WB, Zhang CH, Wang YC. 2011. Extracellular matrix protein 1, a novel prognostic factor, is associated with metastatic potential of hepatocellular carcinoma. Med Oncol 28 Suppl 1; S318-25. doi: 10.1007/s12032-010-9763-1.

Ferlay J, Soerjomataram I, Dikshit R, Eser S, Mathers C, Rebelo M, Parkin DM, Forman D, Bray F. 2015. Cancer incidence and mortality worldwide: sources, methods and major patterns in GLOBOCAN 2012. Int J Cancer 136; E359-86. doi: 10.1002/ijc.29210.

Friard O, Re A, Taverna D, De Bortoli M, Cora D. 2010. CircuitsDB: a database of mixed microRNA/transcription factor feed-forward regulatory circuits in human and mouse. BMC Bioinformatics 11; 435. doi: 10.1186/1471-2105-11-435.

Horvath Z, Kovalszky I, Fullar A, Kiss K, Schaff Z, Iozzo RV, Baghy K. 2014. Decorin deficiency promotes hepatic carcinogenesis. Matrix Biol 35; 194-205. doi: 10.1016/j.matbio.2013.11.004.

Jeggari A, Marks DS, Larsson E. 2012. miRcode: a map of putative microRNA target sites in the long non-coding transcriptome. Bioinformatics 28; 2062-3. doi: 10.1093/bioinformatics/bts344.

Klingenberg M, Matsuda A, Diederichs S, Patel T. 2017. Non-coding RNA in hepatocellular carcinoma: Mechanisms, biomarkers and therapeutic targets. J Hepatol 67; 603-618. doi: 10.1016/j.jhep.2017.04.009.

Langfelder P, Horvath S. 2008. WGCNA: an R package for weighted correlation network analysis. BMC Bioinformatics 9; 559. doi: 10.1186/1471-2105-9-559.

Li W, Xie L, He X, Li J, Tu K, Wei L, Wu J, Guo Y, Ma X, Zhang P, Pan Z, Hu X, Zhao Y, Xie H, Jiang G, Chen T, Wang J, Zheng S, Cheng J, Wan D, Yang S, Li Y, Gu J. 2008. Diagnostic and prognostic implications of microRNAs in human hepatocellular carcinoma. Int J Cancer 123; 1616-22. doi: 10.1002/ijc.23693.

Liang X, Zhao Q, Geng T, Luo S, He Q. 2018. MiR-106b regulates the apoptosis and tumorigenesis of hepatocellular carcinoma via targeting Zinc finger and BTB domain-containing protein 7A (Zbtb7a). J Biochem Mol Toxicol 32; e22169. doi: 10.1002/jbt.22169.

Lim HY, Sohn I, Deng S, Lee J, Jung SH, Mao M, Xu J, Wang K, Shi S, Joh JW, Choi YL, Park CK. 2013. Prediction of disease-free survival in hepatocellular carcinoma by gene expression profiling. Ann Surg Oncol 20; 374753. doi: 10.1245/s10434-013-3070-y.

Peer] reviewing PDF | (2019:10:42157:1:1:NEW 7 Mar 2020) 
Lu Y, Yue X, Cui Y, Zhang J, Wang K. 2013. MicroRNA-124 suppresses growth of human hepatocellular carcinoma by targeting STAT3. Biochem Biophys Res Commun 441; 873-9. doi: 10.1016/j.bbrc.2013.10.157.

Nault JC, Sutter O, Nahon P, Ganne-Carrie N, Seror O. 2018. Percutaneous treatment of hepatocellular carcinoma: State of the art and innovations. J Hepatol 68; 783-797. doi: 10.1016/j.jhep.2017.10.004.

Ohrnberger S, Thavamani A, Braeuning A, Lipka DB, Kirilov M, Geffers R, Autenrieth SE, Romer M, Zell A, Bonin M, Schwarz M, Schutz G, Schirmacher P, Plass C, Longerich T, Nordheim A. 2015. Dysregulated serum response factor triggers formation of hepatocellular carcinoma. Hepatology 61; 979-89. doi: 10.1002/hep.27539.

Ren F, Li J, Wang Y, Wang Y, Feng S, Yuan Z, Qian X. 2018. The Effects of Angelica Sinensis Polysaccharide on Tumor Growth and Iron Metabolism by Regulating Hepcidin in Tumor-Bearing Mice. Cell Physiol Biochem 47; 1084-1094. doi: 10.1159/000490185.

Shankaran V, Ikeda H, Bruce AT, White JM, Swanson PE, Old LJ, Schreiber RD. 2001. IFNgamma and lymphocytes prevent primary tumour development and shape tumour immunogenicity. Nature 410; 1107-11. doi: $10.1038 / 35074122$.

Shannon P, Markiel A, Ozier O, Baliga NS, Wang JT, Ramage D, Amin N, Schwikowski B, Ideker T. 2003. Cytoscape: a software environment for integrated models of biomolecular interaction networks. Genome Res 13; 2498-504. doi: 10.1101/gr.1239303.

Shih TC, Tien YJ, Wen CJ, Yeh TS, Yu MC, Huang CH, Lee YS, Yen TC, Hsieh SY. 2012. MicroRNA-214 downregulation contributes to tumor angiogenesis by inducing secretion of the hepatoma-derived growth factor in human hepatoma. $J$ Hepatol 57; 584-91. doi: 10.1016/j.jhep.2012.04.031.

Sun H, Song J, Weng C, Xu J, Huang M, Huang Q, Sun R, Xiao W, Sun C. 2017. Association of decreased expression of the macrophage scavenger receptor MARCO with tumor progression and poor prognosis in human hepatocellular carcinoma. J Gastroenterol Hepatol 32; 1107-1114. doi: 10.1111/jgh.13633.

Wang H, Luo J, Liu C, Niu H, Wang J, Liu Q, Zhao Z, Xu H, Ding Y, Sun J, Zhang Q. 2017. Investigating MicroRNA and transcription factor co-regulatory networks in colorectal cancer. BMC Bioinformatics 18; 388. doi: 10.1186/s12859-017-1796-4.

Wang M, Zhang J, Tong L, Ma X, Qiu X. 2015. MiR-195 is a key negative regulator of hepatocellular carcinoma metastasis by targeting FGF2 and VEGFA. Int J Clin Exp Pathol 8; 14110-20. doi:

Yang H, Kang K, Cheng C, Mamillapalli R, Taylor HS. 2015. Integrative Analysis Reveals Regulatory Programs in Endometriosis. Reprod Sci 22; 1060-72. doi: 10.1177/1933719115592709.

Yin L, Cai Z, Zhu B, Xu C. 2018. Identification of Key Pathways and Genes in the Dynamic Progression of HCC Based on WGCNA. Genes (Basel) 9. doi: 10.3390/genes9020092.

Zhao L, Gu C, Ye M, Zhang Z, Li L, Fan W, Meng Y. 2018. Integration analysis of microRNA and mRNA paired expression profiling identifies deregulated microRNA-transcription factor-gene regulatory networks in ovarian endometriosis. Reprod Biol Endocrinol 16; 4. doi: 10.1186/s12958-017-0319-5.

Peer] reviewing PDF | (2019:10:42157:1:1:NEW 7 Mar 2020) 
Figure 1

The flowchart of identification of key mRNAs and mRNA-interacting miRNAs. 


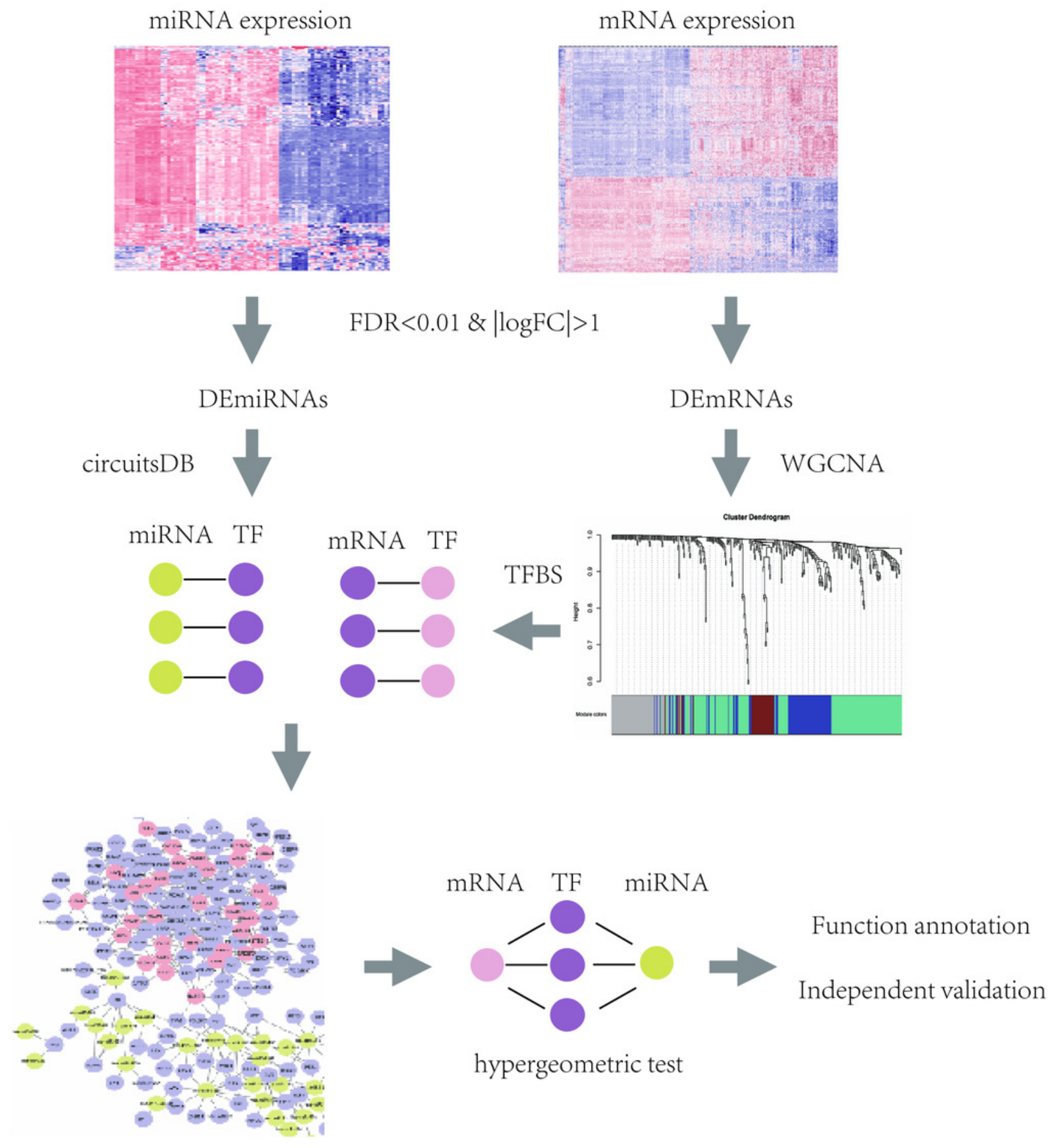


Figure 2

Heatmaps of profiles GSE36376 (A) and GSE36915 (B) from GEO database.

The degree of expression is indicated by different colors, with expression increasing between blue and red. Blue, low expression; red, high expression.

A

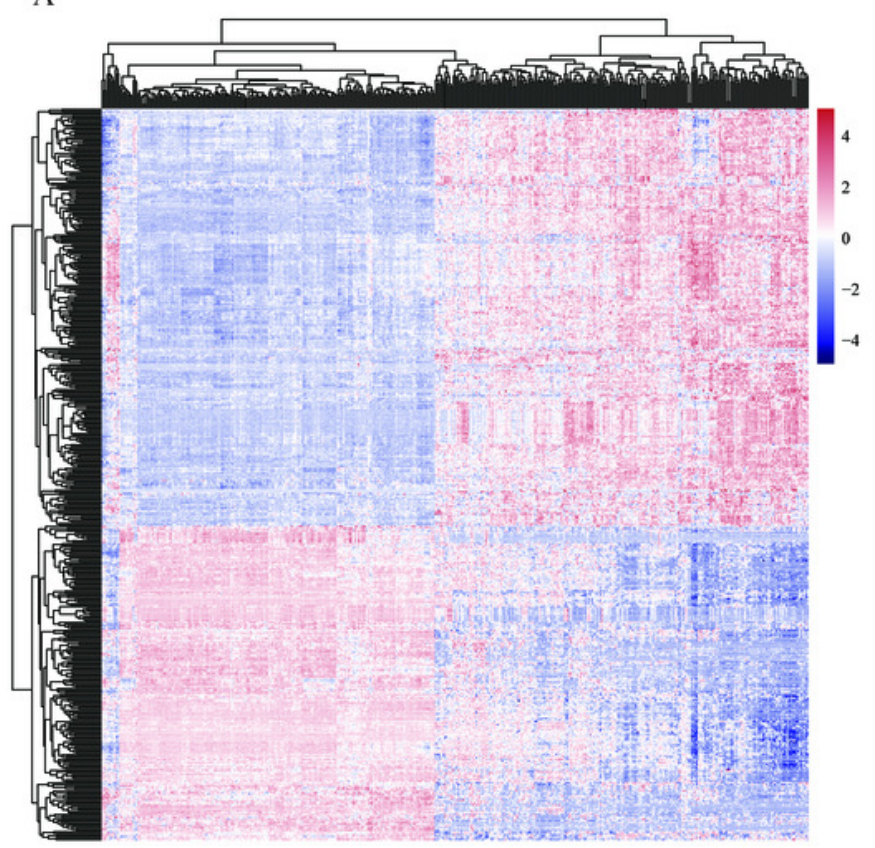

B

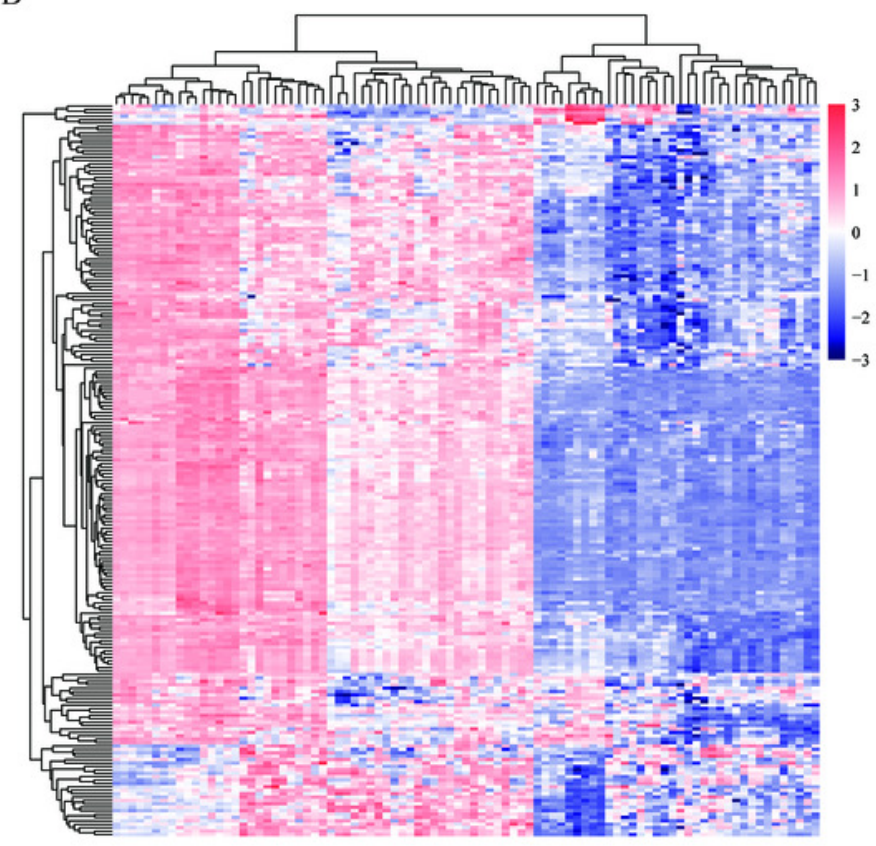




\section{Figure 3}

Construction of WGCNA network.

(A) The distributions of $\log (k)$ and $\log (p(k))$ coefficients and mean connectivity corresponding to thresholds of significance $(\beta)$ were shown. (B) Construction of the system clustering tree based on Pearson's coefficients of DEGs. (C) Correlation between significance and gene expression of brown module membership. (D) Correlation between gene expression in modules and traits.

A

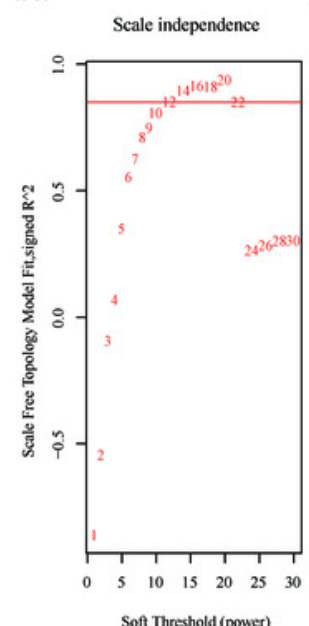

$\mathrm{D}$

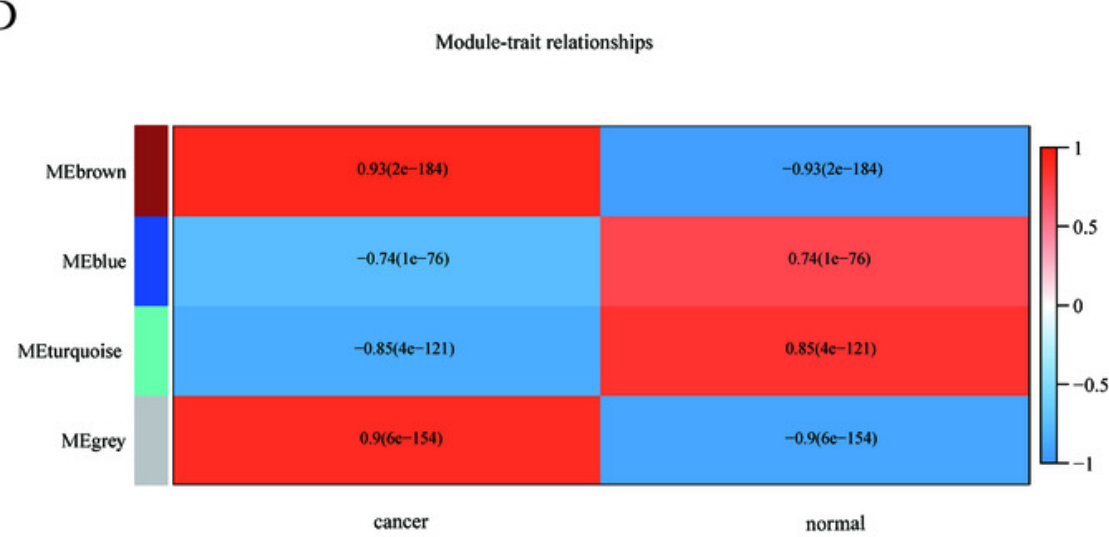

$\mathrm{C}$
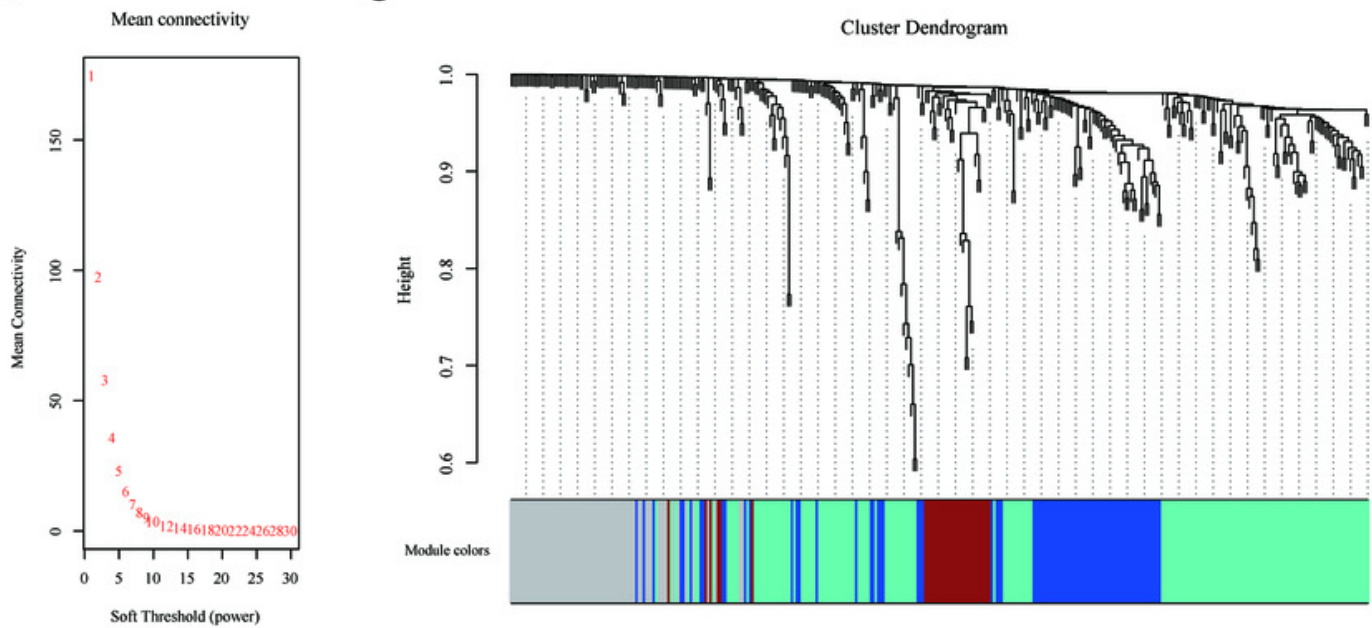

E

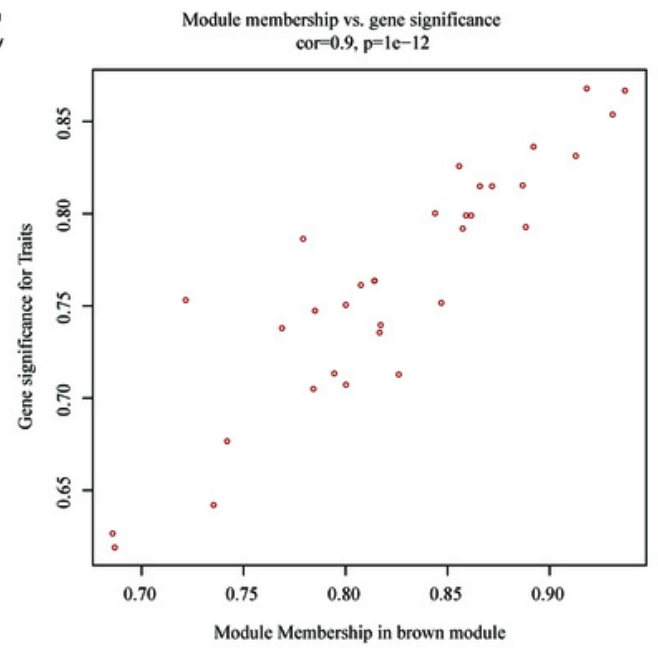


Figure 4

Construction of the mRNA-TF-miRNA triple interaction network.

Each circular represents a gene. The purple circular represents TF, the red circular represents mRNA, and the yellow circular represents miRNA. Their interactions are displayed as solid lines. Core TFs that simultaneously connect with both mRNAs and miRNAs are emphasize with red circles. 


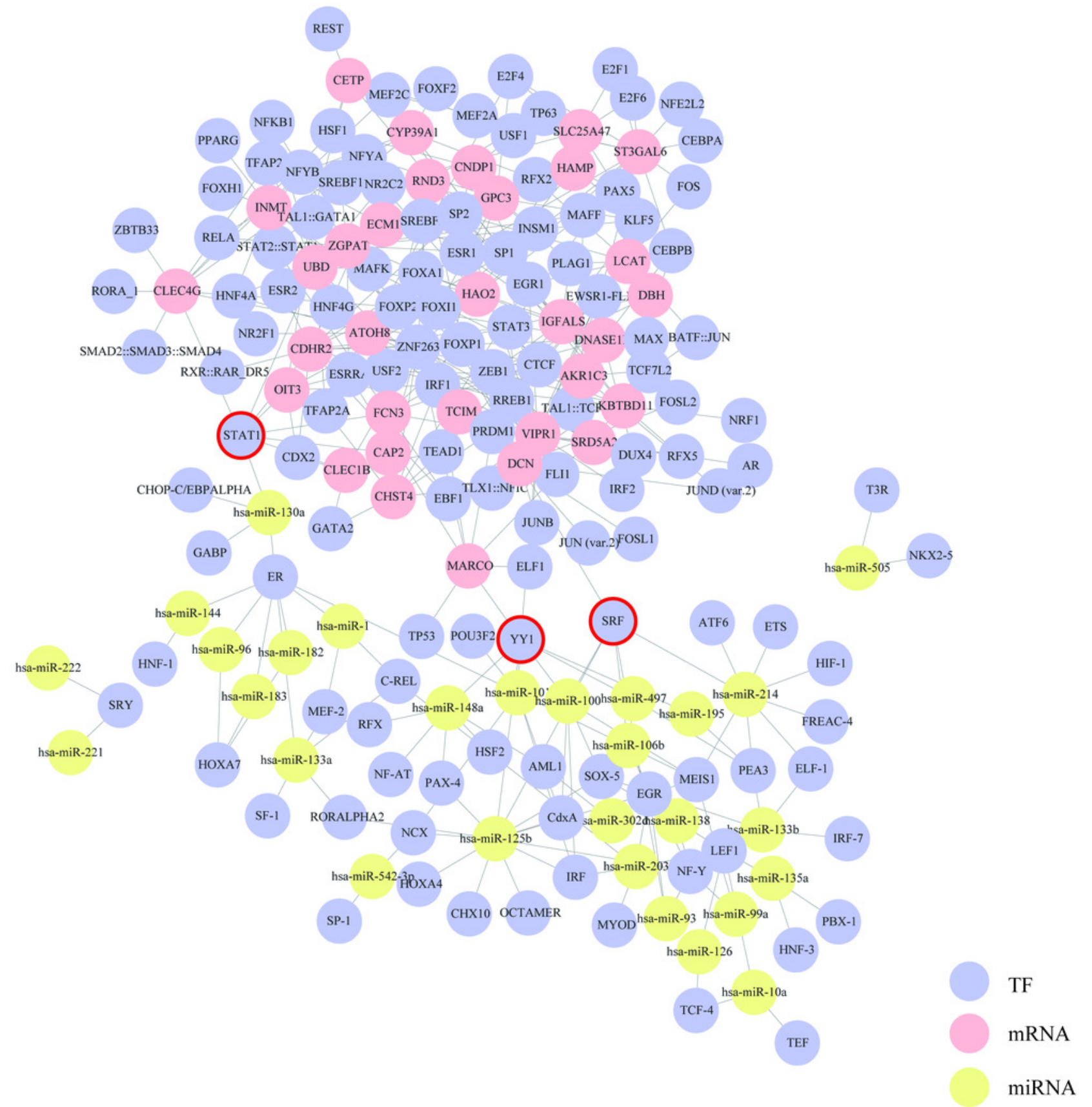


Figure 5

GO-term analysis of hsa-miR-106b and hsa-miR-195.

Cellular component (CC) are shown in red, biological process (BP) are shown in blue, and molecular function (MF) are shown in green. Description of GO-term are listed on the left.

A

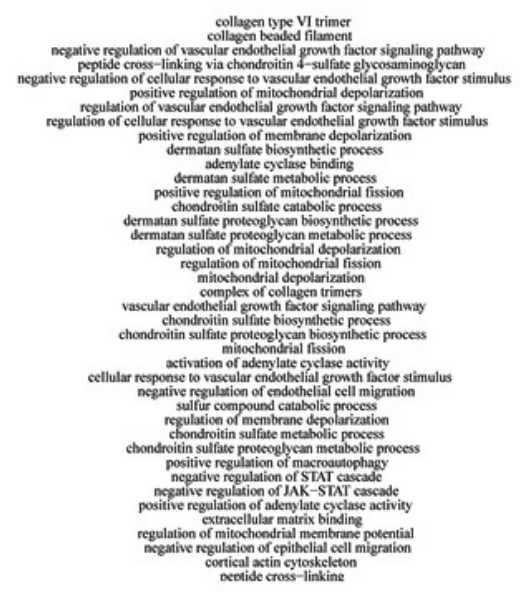

B

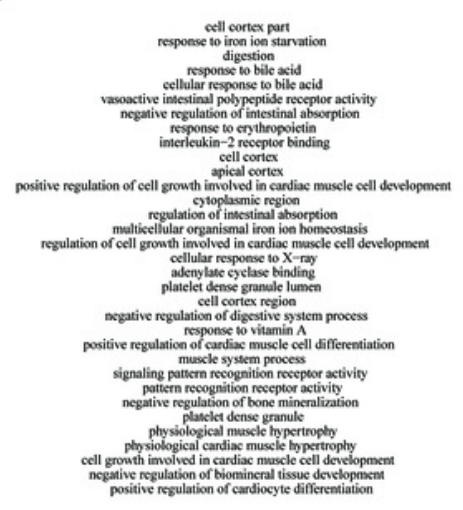

hsa-miR-106b

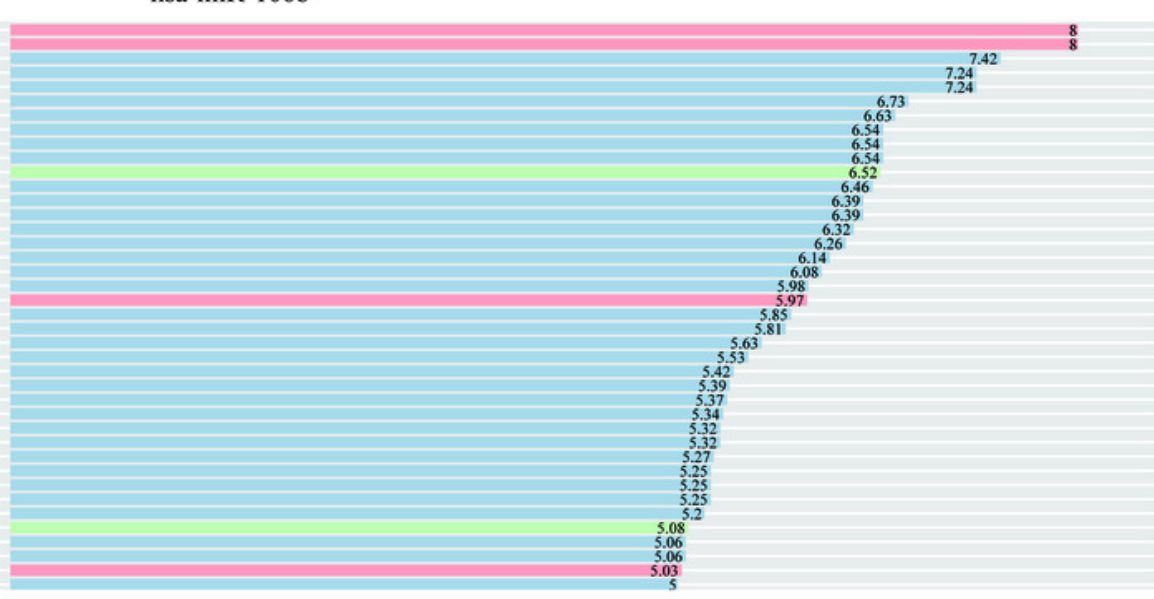

hsa-miR-195

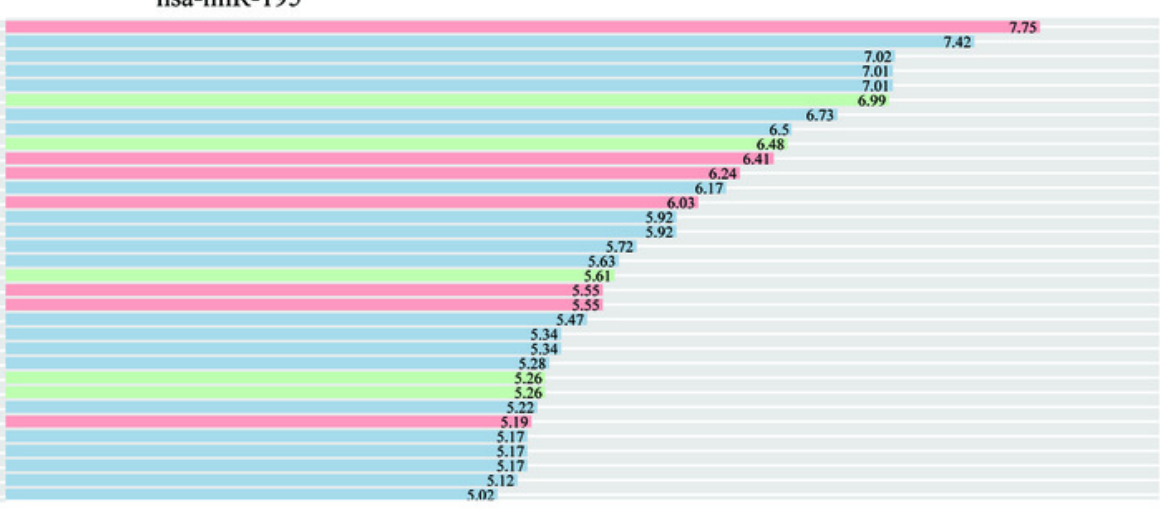


Figure 6

The hierarchical network diagram of GO-term analysis of hsa-miR-106b and hsamiR-195.

The hollow circulars are GO terms. GO terms with high significance are colored with yellow.

A

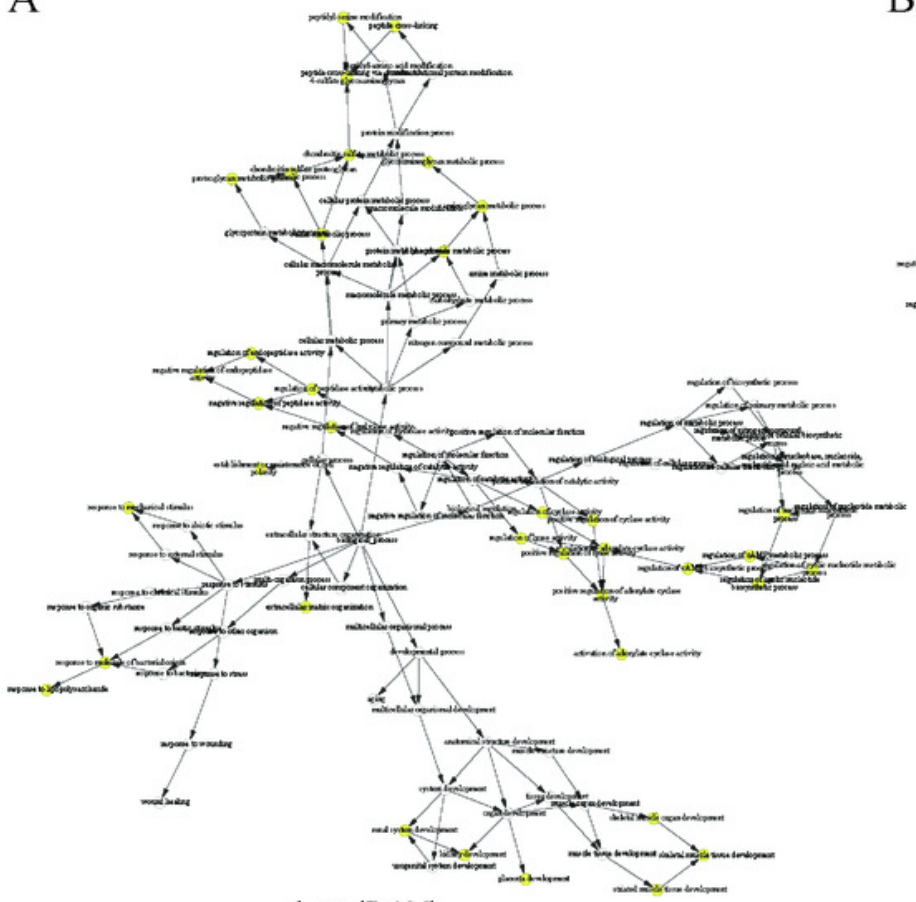

hsa-miR-106b
B

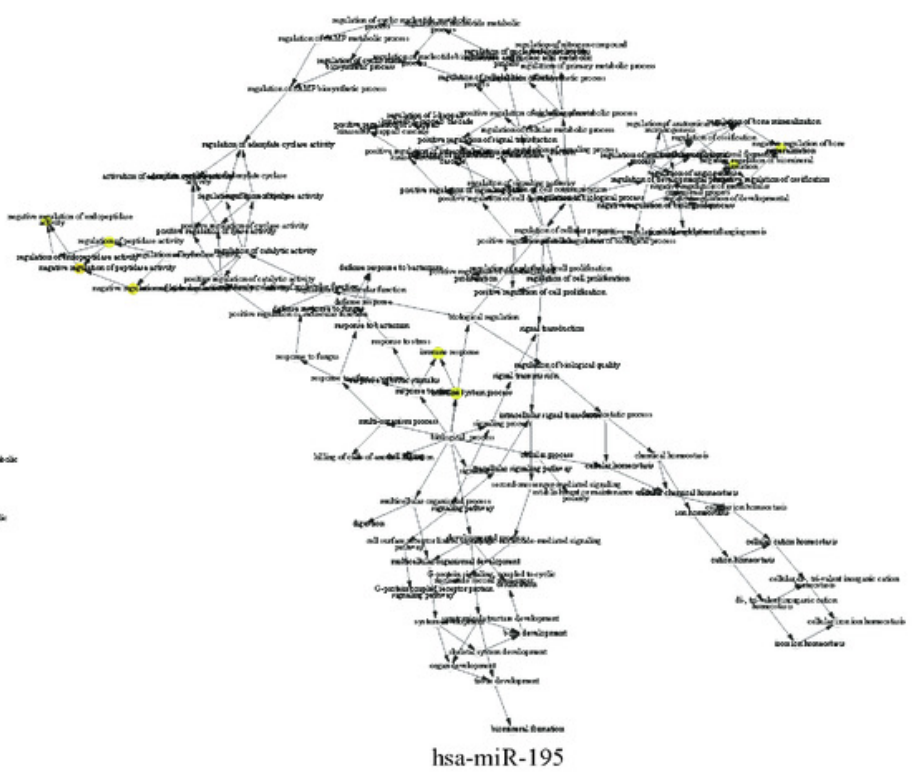


Table $\mathbf{1}$ (on next page)

List of miRNA-mRNA interactions. 
Table 1:

List of miRNA-mRNA interactions.

\begin{tabular}{|c|c|c|c|c|c|c|c|c|c|c|c|c|}
\hline miRNA & mRNA & & & & & & & & & & & \\
\hline hsa-miR-148a & MARCO & TCIM & & & & & & & & & & \\
\hline hsa-miR-302d & CAP2 & ECM1 & HAMP & TCIM & & & & & & & & \\
\hline has-miR-1 & CAP2 & TCIM & & & & & & & & & & \\
\hline hsa-miR-133a & TCIM & & & & & & & & & & & \\
\hline hsa-miR-130a & CAP2 & FCN3 & UBD & OIT3 & CLEC1B & TCIM & CLEC4G & & & & & \\
\hline hsa-miR-93 & $\mathrm{DCN}$ & CAP2 & TCIM & & & & & & & & & \\
\hline hsa-miR-106b & $\mathrm{DCN}$ & CAP2 & TCIM & & & & & & & & & \\
\hline hsa-miR-133b & TCIM & & & & & & & & & & & \\
\hline hsa-miR-96 & CAP2 & ECM1 & HAMP & TCIM & & & & & & & & \\
\hline hsa-miR-144 & CAP2 & ECM1 & HAMP & TCIM & & & & & & & & \\
\hline hsa-miR-183 & CAP2 & ECM1 & HAMP & TCIM & & & & & & & & \\
\hline hsa-miR-182 & CAP2 & ECM1 & HAMP & TCIM & & & & & & & & \\
\hline hsa-miR-135a & CAP2 & TCIM & & & & & & & & & & \\
\hline hsa-miR-138 & CAP2 & TCIM & & & & & & & & & & \\
\hline hsa-miR-542-3p & CAP2 & ECM1 & HAMP & TCIM & & & & & & & & \\
\hline hsa-miR-497 & VIPR1 & CAP2 & ECM1 & MARCO & HAMP & TCIM & & & & & & \\
\hline hsa-miR-195 & VIPR1 & CAP2 & ECM1 & MARCO & HAMP & TCIM & & & & & & \\
\hline hsa-miR-222 & DBH & CAP2 & RND3 & ECM1 & HAO2 & CNDP1 & CETP & OIT3 & CLEC1B & SRD5A2 & HAMP & TCI \\
\hline has-miR-221 & $\mathrm{DBH}$ & CAP2 & RND3 & ECM1 & $\mathrm{HAO} 2$ & CNDP1 & CETP & OIT3 & CLEC1B & SRD5A2 & HAMP & TCI \\
\hline hsa-miR-10a & CAP2 & TCIM & & & & & & & & & & \\
\hline hsa-miR-203 & TCIM & & & & & & & & & & & \\
\hline hsa-miR-100 & MARCO & TCIM & & & & & & & & & & \\
\hline hsa-miR-126 & CAP2 & ECM1 & HAMP & TCIM & & & & & & & & \\
\hline hsa-miR-99a & CAP2 & ECM1 & HAMP & TCIM & & & & & & & & \\
\hline hsa-miR-505 & CAP2 & ECM1 & HAMP & TCIM & $\square$ & $\square$ & $\square$ & $\square$ & $\square$ & $\square$ & $\square$ & $\square$ \\
\hline
\end{tabular}

